\title{
CORRIGENDUM
}

doi:10.1038/nature05086

\section{Structure of the $E$. coli protein-conducting channel bound to a translating ribosome}

Kakoli Mitra, Christiane Schaffitzel, Tanvir Shaikh, Florence Tama, Simon Jenni, Charles L. Brooks, Nenad Ban \& Joachim Frank

Nature 438, 318-324 (2005)

We wish to withdraw a poorly worded criticism of Arthur E. Johnson's fluorescent studies of the ribosome-PCC complex, contained in the Supplementary Information to this Article. The issues raised by the discrepancies between several cryo-EM studies and the body of work from Johnson's group regarding solvent accessibility of the ribosome-PCC junction warrant more extensive study and discussion in the scientific community. The withdrawal of the statement has no bearing on the results of our study or on their interpretation. 